\title{
Development and evaluation of a system to assess the effect of footwear on the in shoe plantar pressure and shear during gait
}

\author{
Manabu Takano ${ }^{1 *}$, Hiroshi Noguchi ${ }^{1}$, Makoto Oe ${ }^{2}$, Hiromi Sanada ${ }^{3}$ and Taketoshi Mori ${ }^{1}$
}

\begin{abstract}
In this paper, we propose a system to assess the effect of footwear on the in-shoe plantar pressure and shear during gait. In our previous study, we developed a system for measuring the plantar pressure and shear inside footwear using specialized shoes and insoles with sensors. In this study, based on the previous study, 1) we realized a method to measure directly the in-shoe plantar pressure and shear applied from footwear, 2) verified the validity and reliability of the method and 3) evaluated the ability of the system to assess the effect of footwear by measurements with multiple subjects and various footwear. The reliability and validity were demonstrated by comparison with our previous system. From the evaluation of the newly developed system, we found four suggestions as the following; a) Footwear does not always reduce the pressure and shear force. b) Fixing the instep with shoelace or straps is effective in the reduction of shear force on risk regions of plantar. c) People with callus show significantly higher peak shear force $(3.31 \pm 0.78(\mathrm{kgf}))$ than people without callus $(2.03 \pm 0.42(\mathrm{kgf}))$ when they walk with their usual wearing shoes $(p<0.01)$. But in barefoot walking, there is no significant difference $(2.59 \pm 1.08$ (kgf) versus $2.67 \pm$ 0.99 (kgf), $(p=0.66))$. d) People with callus can reduce the shear force by changing footwear. The system employed in this study can be used for assessing the effect of the intervention such as footwear or instruction of a walk.
\end{abstract}

Keywords: Callosity; Diabetic foot; Gait analysis; Direct measurement; Therapeutic shoes

\section{Background}

Gait has been widely and variously analyzed in clinical settings [1]. For example, gait parameters such as walking speed, cadence, step length and plantar pressure have been measured for fall [2], diabetic foot [3], and osteoarthritis [4]. Intervention by footwear is one of the major methods for improving gait parameters for both prevention and therapy. However, the evaluation of intervention by footwear [5-7] is difficult, especially for diabetic patients because they sometimes have neuropathy or foot deformity $[8,9]$. Because their sensitivity for pressure and shear force has decreased by neuropathy, it is hard to complain to clinicians about the pain or abnormality on their plantar. Also, even if patients have foot deformity, clinicians have been estimating the effect of footwear only experientially.

\footnotetext{
* Correspondence: manabutakano-tky@umin.ac.jp

${ }^{1}$ Department of Life Support Technology (Molten), Graduate School of Medicine, The University of Tokyo, Tokyo, Japan

Full list of author information is available at the end of the article
}

Neuropathy, deformity and repetitive stress are known key factors of foot ulcer [10]. Moreover, the forces known to directly act on the foot are the plantar pressure (the force that act in the vertical direction) and the shear force (the shift force in the horizontal direction) [11]. If the patient has foot deformities or callus, the application of high pressure on the local regions would result in ulcer formation through infection or injury [12]. It has been particularly reported that patients with callus have very high risks of developing foot ulceration. Furthermore, the plantar pressure and the shear force are associated with callus formation [13]. To prevent foot ulcer, both consultation with experts and usage of footwear that reduce the force load on local regions are recommended [14]. High pressure is known to be exerted on the regions of the first and second metatarsal heads during gait [15], and the regions are frequent site of callus.

Owing to the lack of standard methods for the objective clinical assessment of whether the footwear is appropriate or not [6], callus formation has not been 
effectively prevented. Hence in many cases, rather than preventing it, therapeutic care is given, to remove the callus after its formation. It is therefore important to evaluate the effect of footwear on the risk plantar regions by directly measuring the pressure and shear force on the regions applied from footwear.

There are two major methods for measuring the force applied on the foot during gait. One involves the use of a force plate or sensors embedded in the ground [16-18]. This method can only be applied in special places, and the measured force is not the force on risk regions of the plantar but the force that the footwear applies on the ground. The other method involves the use of specialized shoes or insoles with embedded sensors $[19,20]$. In a study of Lord $M$ et al., thin pressure sensor sheets and special insoles with embedded 3.8mm-thick shear stress sensors were used [19]. It is also difficult to use the insoles of the subject when applying this method, because the sensors are embedded in special insoles that are part of the footwear.

In a previous study conducted in our laboratory [21], a system for the simultaneous measurement of the shear force and pressure distribution during gait was developed. The system use the same pressure sensor sheet in the previous study [19] mentioned above as well as novel 1.2-mm-thick shear force sensors embedded in 7-mmthick specialized insoles. Because thin and soft sensors were used in the system, measurements could be safely taken in clinical settings. However the system also uses specialized insoles for the measurement purpose and can therefore not be used to directly measure the in-shoe pressure and shear on the plantar using usual footwear. Furthermore, this method actually involves the measurement of the force that the foot applies on the footwear and not the direct measurement of the force that the footwear applies on the plantar risk regions. Hence, based on our previous system [21] whose safeness in clinical settings has been shown, we developed another new system for directly measuring the plantar pressure and shear applied from footwear.

The purpose of this study was the development and evaluation of a system for assessing the effect of footwear on the direct in-shoe plantar pressure and shear force during gait. The study consisted of three parts.

1) The realization and investigation of a method which seems best for simultaneously measuring the direct in-shoe plantar pressure and shear using various footwear.

Since there are sensor sheets that are known to be reliable and valid for measuring the plantar pressure $[22,23]$ especially we concentrate on investigating the possible measuring method of the shear force in conjunction with the pressure sensor sheets. Based on our previous system [21], which is safe for clinical settings, we developed a method for integrating the sensor with the foot plantar to measure the force applied to the plantar and enable the use of various footwear. To maintain the plantar condition, we embedded sensors in thin sheets with elasticity and friction coefficients similar to those of the plantar.

2) Verification of the validity and reliability of the method To verify the reliability of the developed method, we examined the stability of the measured values during a trial and between trials. To verify the criteria-related validity, we compared the measured values with those obtained using the previous system. To assess the content validity, we examined the possibility of detecting the difference between the measured values for different subjects. We also verified whether the measured values decreased as expected when using a footwear that reduces the pressure and shear.

3) Evaluation of the ability of the system to assess the effect of footwear on the in-shoe plantar pressure and shear during gait

We evaluated the system as an assessment method of the effect of footwear intervention. Considering the typical intervention by footwear, we examined whether subjects with callus could reduce the pressure and shear by changing their footwear. First, we examined the difference between simulated barefoot walking and walking with footwear for multiple subjects. Second, we examined the difference between the measured values for various footwear with different characteristics using a subject with callus.

These three parts are described in Section 2-4. The study protocol was approved by the Ethical Committee of the Graduate School of Medicine, The University of Tokyo (\#10310).

\section{Methods}

\section{Development of a system for measuring the direct}

\section{in-shoe pressure and shear on the plantar during gait}

An overview of the developed system is shown in Figure 1. The system consists of two major parts, namely the part for measuring the pressure distribution (which is beneath the shoes) and the sheet part for measuring the shear force (which is integrated with the plantar). Both the left and right shoes have both parts. Unlike previous studies, which required specialized footwear embedded sensors, these two parts are designed to be independent of the footwear so that the pressure and shear applied directly on the plantar during gait could be measured for different footwear such as shoes, insoles, and socks. The system is designed for continuous measurement of the in-shoe 


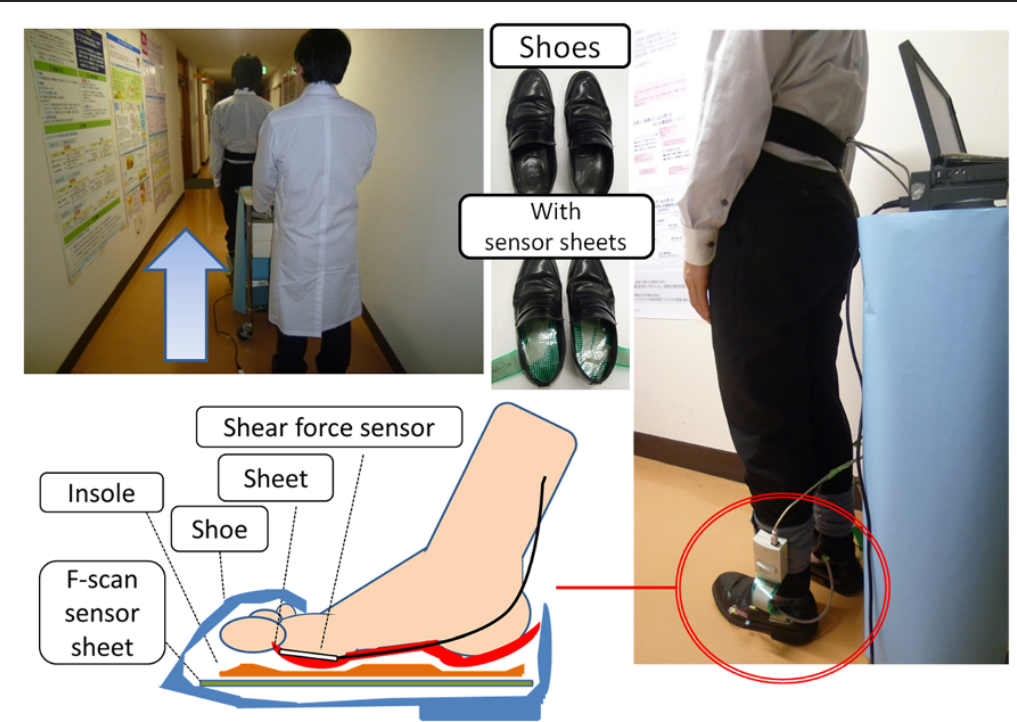

Figure 1 Overview of the system.

pressure and shear acting on the plantar when standing still and during gait.

\section{Sheets for obtaining the pressure distribution}

To obtain the pressure distribution, we use the F-Scan system (Nitta Corporation, Osaka). F-Scan sheets about $0.2 \mathrm{~mm}$ thick were placed between the original bottom of the shoes and the insoles. To ensure fit with different shoes, we prepared F-Scan sheets of various sizes. Because F-Scan sheets are widely used for evaluating the effects of insoles, the pressure distribution can be measured in a similar way with the previous studies $[24,25]$. The pressure distribution data were collected at $50 \mathrm{~Hz}$ using a PC.

\section{Sheet with a shear force sensor}

The footprint, the sheet with an embedded sensor, and one state of the sheet attached to the plantar are shown in Figure 2. To fit the shape of the plantar of the subject, the sheets are made using footprints. Footprints are used in clinical settings for easy examination of the pressure distribution on the plantar when standing still barefoot. The use of footprints enables determination of the region to which high pressure is applied, and hence the determination of the region that should be examined. In this study, we use footprints to identify the first and second metatarsal heads that are under high pressure in the push off phase, and hence the points at which the shear force sensors should be embedded.

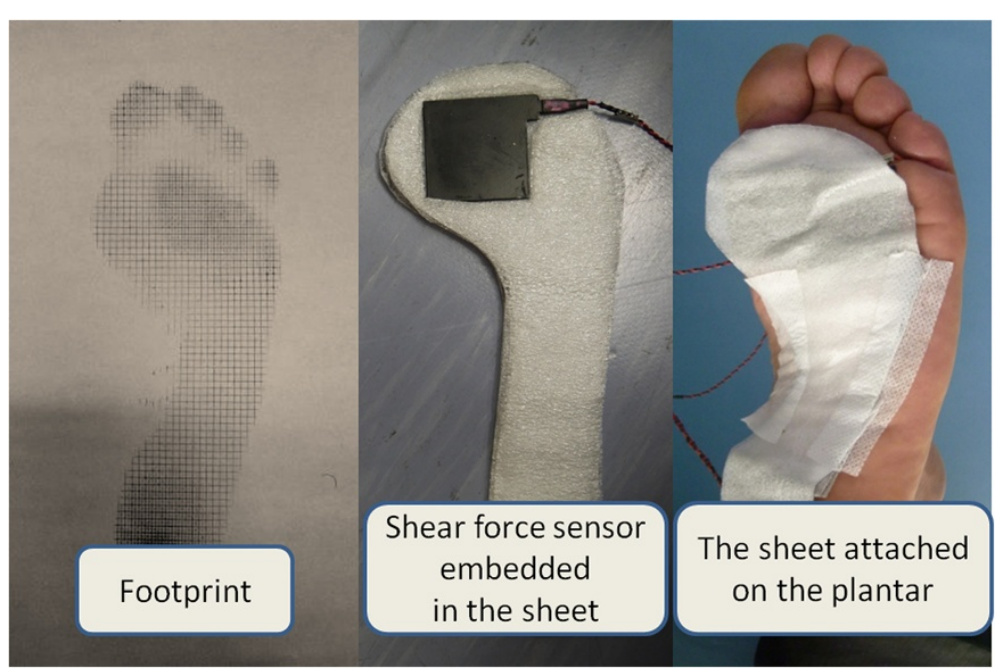

Figure 2 Overview of the shear force sensor and the sheet. 

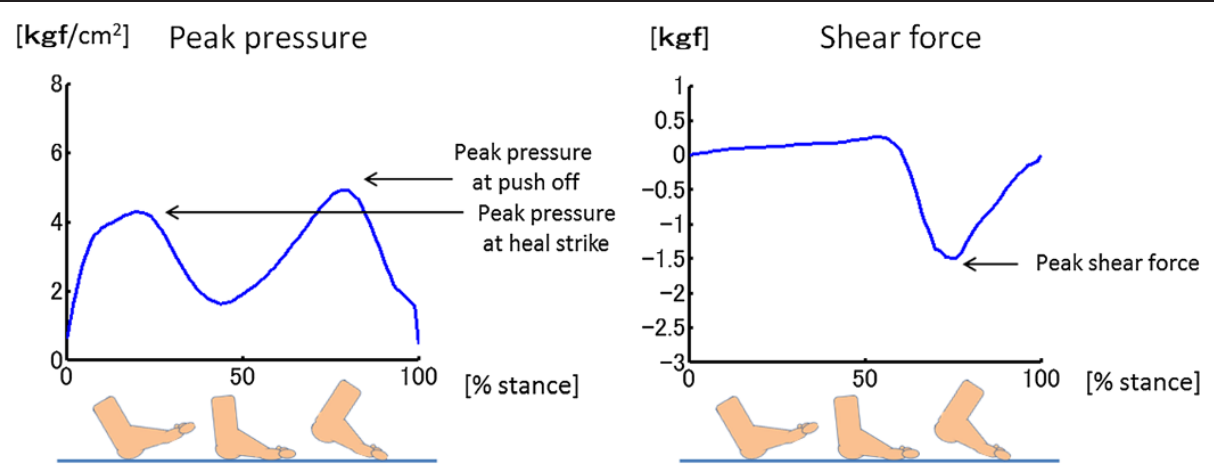

Figure 3 Waveforms of peak pressure and shear force during a stance phase.

The sheets were produced from polyethylene. By stacking three pieces of the polyethylene sheets, we eliminated the difference between the levels of the sheets and the sensor. We designed the sheets to have an overall thickness of $2.6 \mathrm{~mm}$ or less to reduce the effect of the shape of the sensor and to maintain the undulation shape of the plantar. We placed the one-axis shear force sensor (Keisoku Support Ltd., Hiroshima), which measured $35 \times$ $35 \times 1.2\left(\mathrm{~mm}^{3}\right)$ in the region of the first and second metatarsal heads. The sensor had a load-bearing shear force of about $200(\mathrm{~N})$ and resolution of $6.1 \times 10^{-4}(\mathrm{kgf})$. We used medical tape to attach the sheet because it is less invasive and does not shift or peel off during gait.

We collected the data from the shear force sensor at $500 \mathrm{~Hz}$ using a data logger. As shown in Figure 1, the data logger and PC were placed on a caster so that the researcher could move along with the caster as the subject walked.

The pressure distribution data were collected at $50 \mathrm{~Hz}$ and the shear force data were collected at $500 \mathrm{~Hz}$. However, we down-sampled the shear force data to $50 \mathrm{~Hz}$ to use the same frequency as the pressure distribution data. One stance phase is the period which is cut out from the load of the heel begin to take to the load of the toe eliminate by using the value of total load. The stance phase was normalized to $0 \%-100 \%$ with respect to time.

\section{Examination of the measurement}

We examined whether the newly developed system could be used for the measurement. Figure 3 shows the mean peak pressure and the mean shear force for 15 steps of a subject obtained using the new system.

During gait, we could obtain continuous data of the pressure and shear force on the left and right foot without loss of data. During the entire measurement process, there were no adverse events nor complaints of walking difficulty. The tendencies of the results for the left and right foot were the same. For simplicity, only the data for the left foot are shown in Verification of the implemented system and Evaluation of the assessment ability of footwear effect on the plantar.

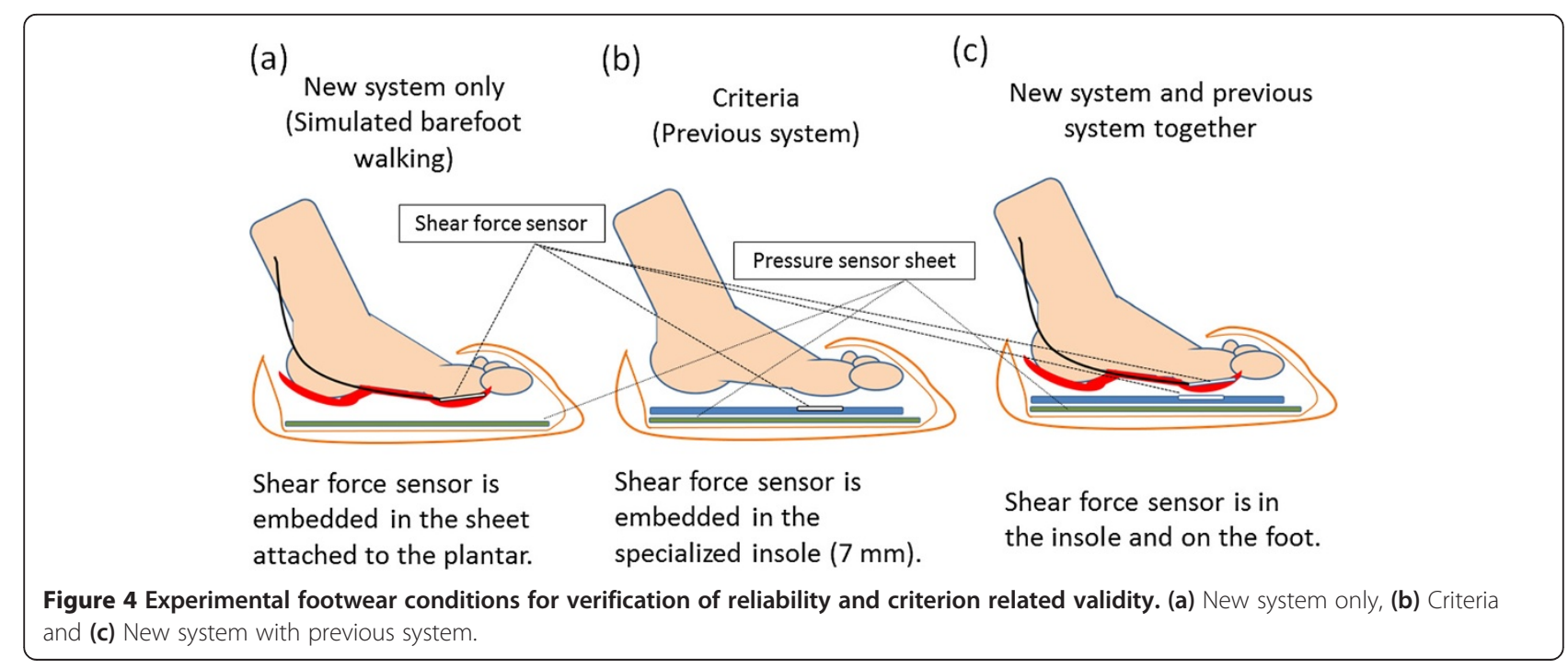




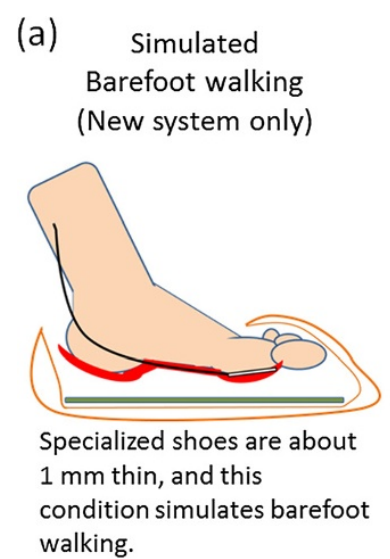

(d)

With soft insoles

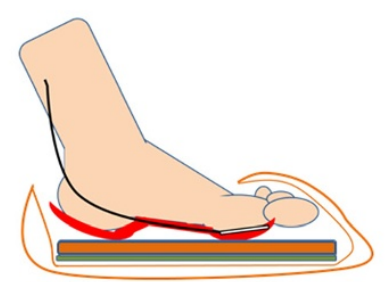

This insole aimed to reduce pressure and shear. (e)

Subject's own footwear

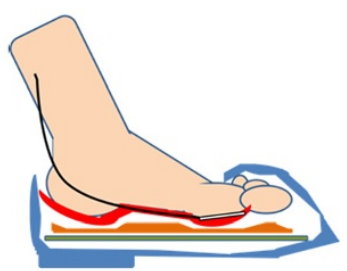

A variety of shoes and insoles are measured. A subject used three types of footwear.

Figure 5 Experimental footwear conditions for verification of content validity and evaluation of this system. (a) Barefoot, (d) With soft insoles and (e) Subject's own footwear.

\section{Verification of the implemented system}

\section{Overview of the verification conditions}

We conducted the verification under the footwear conditions as shown in Figures 4 and 5 . Condition (e) was only used for the evaluation described in Evaluation of the assessment ability of footwear effect on the plantar.

In Figures 4(a) and 5(a), only the newly developed system was used. The pressure distribution-measuring sheet was placed at the bottom of specialized shoes for measurement and the shear force-measuring sheet was attached and unified with the plantar. The specialized shoes used for the measurement were about $1 \mathrm{~mm}$ thick and made from resin. Walking under these conditions

\section{Table 1 Characteristics of the subjects and footwear}

\begin{tabular}{|c|c|c|c|}
\hline & & Mean & (SD) \\
\hline Height in $\mathrm{cm}$ & & 163.1 & $(8.5)$ \\
\hline Weight in kg & & 61.5 & $(10.3)$ \\
\hline BMl in $\mathrm{kg} / \mathrm{m}^{\wedge} 2$ & & 23.1 & $(3.0)$ \\
\hline \multirow[t]{2}{*}{ Sex, n (\%) } & Male & 4 & $(40)$ \\
\hline & Female & 6 & $(60)$ \\
\hline Age in years & & & $(10.9)$ \\
\hline \multirow[t]{2}{*}{ Callus, n (\%) } & Yes & 3 & $(30)$ \\
\hline & No & 7 & $(70)$ \\
\hline Left foot length (sitting) in cm & & 23.4 & $(1.3)$ \\
\hline Right foot length (sitting) in $\mathrm{cm}$ & & 23.5 & $(1.4)$ \\
\hline Left foot length (standing) in $\mathrm{cm}$ & & 23.9 & $(1.5)$ \\
\hline Right foot length (standing) in cm & & 23.9 & $(1.3)$ \\
\hline Shoe size in $\mathrm{cm}$ & & 24.4 & $(1.7)$ \\
\hline Heel hardness in durometer & & 48.5 & $(18.7)$ \\
\hline Shoes weight in $\mathrm{g}$ & & 305 & $(208)$ \\
\hline \multirow[t]{2}{*}{ Heel height in $\mathrm{cm}$} & & 3.3 & $(2.1)$ \\
\hline & & & $N=10$ \\
\hline
\end{tabular}

simulated barefoot walking because the toe and instep were fixed by Velcro without leaving a gap.

Under the condition shown in Figure 4(b), the system developed in the previous study was used. The shoes were the same specialized ones used in (a). The specialized 7-mm-thick insoles for measurement purpose were placed on the pressure distribution-measuring sheets. The shear force sensor was embedded in the specialized insole.

Under the condition shown in Figure 4(c), the abovementioned conditions of (a) and (b) were combined. The shear force sensors were embedded in the two positions. One was embedded in the sheet attached to the plantar and the other in the specialized insole for measurement. In the following, the positions of the sensors are referred to as "(c) on the foot" and "(c) in the insole," respectively.

Under the condition shown in Figure 5(d), the soft insoles were used with (a).

Under the condition shown in Figure 5(e), the subjects wear their usual footwear. If a particular subject used insoles, then the insoles were used for the measurement.

\section{Subjects and footwear}

Ten healthy adults were selected by the snowball sampling method. The height, weight, sex, and age of the subjects we determined, as well as the presence of callus on the first and second metatarsal heads and the size of the plantar in the sitting and standing positions. We used the Brannock Device (The Brannock Device Co., Inc., New York) to measure the size of the foot. We also recorded the measured value of the inner diameter of the shoe length, height of the heel, weight of the shoes, and hardness of the heel region. Height of the heel is the height between the ground and the in-shoe contact area with the heel. There is no shoe whose height of the sole except the heel is very high or low. A durometer 
(Elastron, Inc., Kyoto) was used to measure the hardness of the in-shoe heel region. The characteristics of the subjects and footwear of this study are presented in Table 1.

\section{Measurement procedure}

The subjects walked under the five footwear conditions listed above. After practicing each of the walks for few minutes until the subjects got used to the footwear, they were required to take about 20 steps at a self-selected speed along a 20-m hallway starting from the still standing position. To obtain stable values, the subjects walked five times under each condition as was done in previous studies [26,27]. In the following, we refer to the 20 steps as a trial, and the five trials under the same condition as a set. The subjects were allowed to rest for more than one minute between trials and more than five minutes between sets.

\section{Measurement parameters and data analysis}

To eliminate the effects of acceleration and deceleration, we used 10 intermediate steps of a trial (five steps of left foot and five steps of right foot), excluding at least the first three steps and the last three steps. We used the data of three trials in which there was no loss of data and that had similar stance time.

The peak pressure is the highest value in the pressure distribution at a point in stance phase. A positive measured value of the shear force indicates a forward force whereas a negative value indicates a backward force. The large peak pressure occurred in the heel strike and push off phase. The peak value of the shear force also occurred in the push off phase. High shear force and pressure are risk factors of foot problems such as ulcer. Hence, we attempted to reduce the maximum value of peak pressure or peak shear force by changing the footwear to reduce the in-shoe pressure or shear force acting directly on the plantar.

We used a visual analog scale (VAS) to determine the comfortableness of wearing or walking with the footwear under each condition [28]. The VAS score was scaled from "not comfortable at all (0)" to "most comfortable [10]." We also determined the impressions of the subjects based on their objective descriptions after walking.
We used MATLAB 2012a (Math Works) for the analysis. We considered $\mathrm{p}<0.05$ as a significant difference.

\section{Verification procedure}

The reliability and validity of the system were examined using the following process.

- The values measured during a trial were stable and better than those obtained by the previous system (reliability)

- The values measured during a trial and between trials were similar to those obtained by the previous system (criteria-related validity)

As shown in Figure 4, we examined whether the mean value and standard deviation (SD) of the measurements during a trial under the condition (c) combination of previous system and newly developed one were similar to those obtained (b) previous system as the criteria.

The means and SDs of the peak pressure at heel strike, the peak pressure at push off, and the peak shear force of one trial (five steps each) for 10 subjects were used for the comparison.

By a paired t-test for each subject, we examined whether the means and SDs of the (c) combination could be regarded as equivalence or better than (b) the criteria. By comparing the means and SDs, we determined the criteria-related validity and reliability, respectively.

Because the shear force sensors were in the sheet and on the foot in (c) combination, we used a paired $t$-test to compare the means and SDs of the peak shear forces for (b) criteria with that for (c) in the insole, and that for (b) and that for (c) on the foot.

The measured values for (b) previous system as the criteria and those for (c) combination of the systems during a trial are shown in Table 2 .

The mean \pm SD of the peak pressure at heel strike for (b) was $4.06 \pm 0.97\left(\mathrm{kgf} / \mathrm{cm}^{2}\right)$ and that for (c) was $4.17 \pm$ $0.68\left(\mathrm{kgf} / \mathrm{cm}^{2}\right)$. The mean $\pm \mathrm{SD}$ of the peak pressure at push off for (b) was $4.36 \pm 1.89\left(\mathrm{kgf} / \mathrm{cm}^{2}\right)$ and that for (c) was $4.50 \pm 1.52\left(\mathrm{kgf} / \mathrm{cm}^{2}\right)$. There were no significant differences $(p=0.67, p=0.65)$. The mean \pm SD of the peak shear force for (b) was $1.34 \pm 0.87(\mathrm{kgf})$, that for (c) in the insole was $1.23 \pm 0.63(\mathrm{kgf})$, and that for (c) on the foot was $1.88 \pm 1.90(\mathrm{kgf})$. There were no significant differences $(p=0.75, p=0.12)$. These results show that

Table 2 Reliability and validity during a trial/between trials

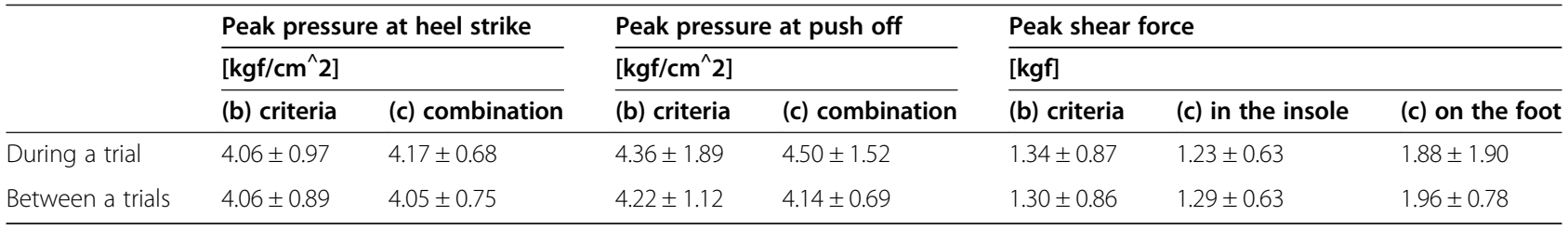


the new system could be used to obtain the measured values as well as those of the previous system.

The mean of the SDs of the peak pressures at heel strike of the 10 subjects for a trial using (b) criteria was 0.33 and that for (c) combination was 0.33 . The mean of the peak pressures at push off for (b) criteria was 0.39 and that for (c) combination was 0.36 . There were no significant differences $(\mathrm{p}=0.57, \mathrm{p}=0.86)$. The mean of the SDs of the peak shear forces of 10 subjects for a trial using (b) criteria was 0.17 , that for (c) in the insole was 0.16 , and that for $(\mathrm{c})$ on the foot was 0.25 . There were no significant differences $(\mathrm{p}=0.67, \mathrm{p}=0.36)$. These results show that the reliability of the new system during a trial is equivalent to that of the previous system.

- The values measured between trials (reliability and criteria-related validity)

We used the means and SDs of the peak pressure at heel strike, peak pressure at push off, and peak shear force of the three mean values (mean of one trial $\times$ three trials).

Using the same method described above, we confirmed that the means and SDs were similar to those of the previous system between trials by using the values for (b) previous system as the criteria and those for (c) combination.

By comparing the means and SDs using a paired t-test for subjects, we verified the criteria-related validity and the reliability, respectively.

The measured values for (b) previous system as the criteria and those for (c) combination of the systems for a set are shown in Table 2.

The mean \pm SD of the peak pressure at heel strike for (b) was $4.06 \pm 0.89\left(\mathrm{kgf} / \mathrm{cm}^{2}\right)$ and that for (c) was $4.05 \pm$ $0.75\left(\mathrm{kgf} / \mathrm{cm}^{2}\right)$. The mean \pm SD of the peak pressure at push off for (b) was $4.22 \pm 1.12\left(\mathrm{kgf} / \mathrm{cm}^{2}\right)$ and that for (c) was $4.14 \pm 0.69\left(\mathrm{kgf} / \mathrm{cm}^{2}\right)$. There were no significant differences $(\mathrm{p}=0.99, \mathrm{p}=0.50)$. The mean $\pm \mathrm{SD}$ of the peak shear force for (b) criteria was $1.30 \pm 0.86$ (kgf), that for (c) in the insole was $1.29 \pm 0.63$ (kgf), and that for (c) on the foot was $1.96 \pm 0.78$ (kgf). Although there was no significant difference between (b) criteria and (c) in the insole ( $p=0.93$ ), (c) on the foot was significantly higher than (b) $(\mathrm{p}=0.02)$. The higher values of the shear force were due to the location of the sensor. The sensor on the sheet was closer to the risk regions than the sensor in the insole since it is directly touching the plantar surface. Hence, the higher values were expected and are admissible.

The mean of the SDs of the peak pressures at heel strike for three trials for (b) was 0.31 and that for (c) was 0.39 . The mean of the peak pressure at push off for (b) was 0.83 and that for (c) was 0.64. There were no significant differences $(p=0.35, p=0.23)$. The mean of the SDs of the peak shear forces for three trials for (b) criteria was 0.12 , that for (c) in the insole was 0.25 , and that for (c) on the foot was 0.18 . There were no significant differences $(\mathrm{p}=0.19, \mathrm{p}=0.51)$.

From here, the newly developed system was used for all the following measurements.

- Could detect the difference between subjects (content validity)

The means and SDs of the peak pressure and peak shear force for 15 steps (five steps $\times$ three trials) for (a) simulated barefoot walking were used for the verification. The data of the 15 steps (five steps $\times$ three trials) were used for all the following verifications and evaluations.

Using the data of the 15 steps for each subject for (a) barefoot, we determined whether the mean of the SDs of the measured values were smaller than the SD between subjects.

Figure 6 shows the peak pressure and peak shear force for 10 subjects under condition (a) simulated barefoot. The mean of the SD of the measured values for 10
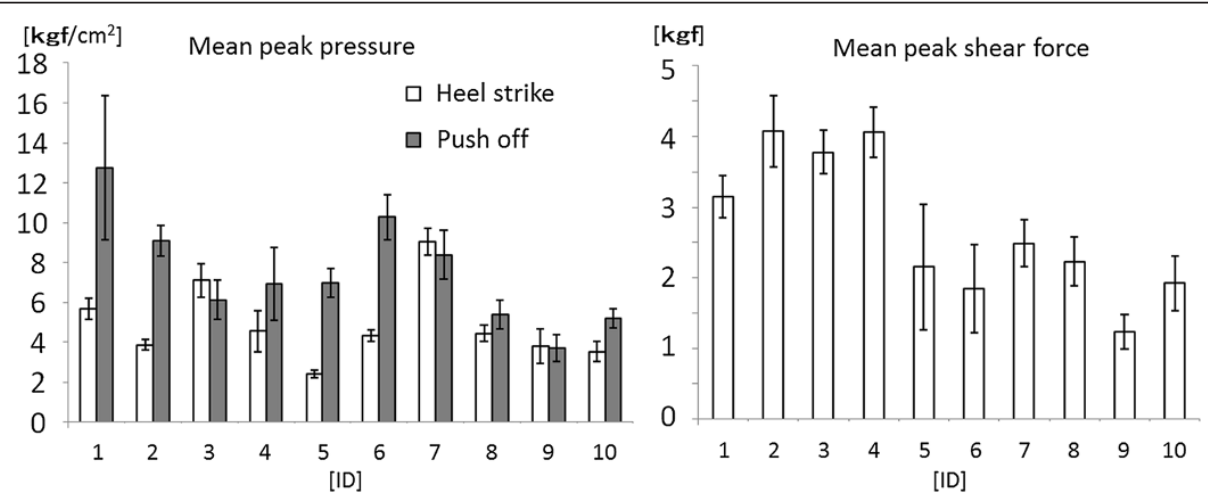

Figure 6 Mean peak pressure and peak shear force for each subject with simulated barefoot walking. 


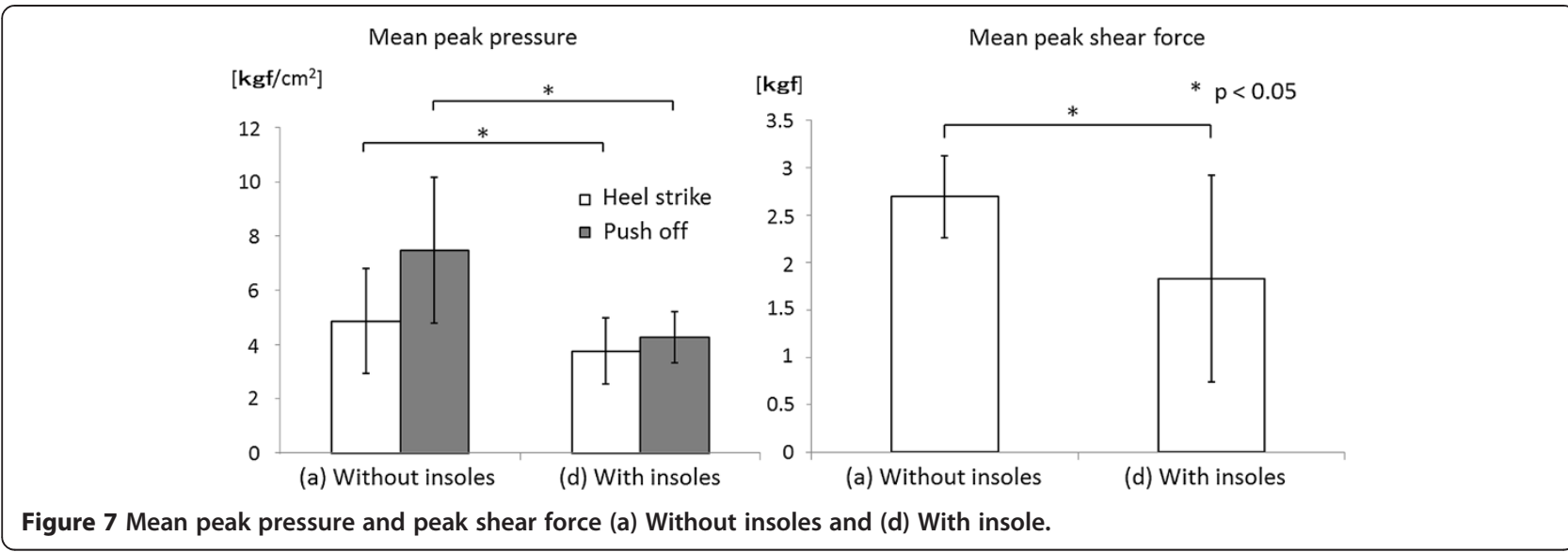

subjects and the SD between the subjects were 0.38 and 0.89 for the peak pressure at heel strike, 0.49 and 1.66 for the peak pressure at push off, and 0.23 and 0.86 for the peak shear force, respectively. Because the mean of the SDs of the measured values were smaller than the SD between the subjects, it was possible to detect the difference between subjects.

- Confirmed the decrease of the load using the insoles, which seemed to reduce the pressure and shear (content validity)

As shown in Figure 5, we compared the measured values of the 10 subjects for (a) barefoot (without insoles) and (d) with insoles.

By a paired t-test for each subject, we confirmed that the pressure and shear force for (d) with insoles were lower than those of (a) without insoles as expected.

Furthermore, we confirmed that the insoles did not largely alter the gait. We calculated the cross-correlation coefficient between the waveforms for (a) without insoles and (d) with insoles, and performed a paired t-test of the stance time. The cross-correlation coefficient was calculated by sliding along the percentage stance.

The mean peak pressure and mean peak shear force for (a) simulated barefoot (without insoles) and (d) using 1 -mm-thick soft (20 durometers) insoles are shown in Figure 7.

For (a) without insoles (barefoot), the mean \pm SD of the peak pressure at heel strike and the peak shear force at push off were $4.86 \pm 1.94(\mathrm{kgf} / \mathrm{cm} 2)$ and $7.47 \pm 2.70$ $(\mathrm{kgf} / \mathrm{cm} 2)$, respectively. Those for $(\mathrm{d})$ with insoles were $3.75 \pm 1.22(\mathrm{kgf} / \mathrm{cm} 2)$ and $4.26 \pm 0.96(\mathrm{kgf} / \mathrm{cm} 2)$, respectively. The insoles significantly reduced the peak pressures $(p=0.004, p<0.001)$. Similar to the above, the mean \pm SD of the peak shear force for (a) without insoles was $2.69 \pm 0.43(\mathrm{kgf})$ and that for (d) with insoles was $1.82 \pm 1.09$ (kgf). The insoles significantly reduced the peak shear force $(\mathrm{p}<0.001)$.
For the pressure, the mean $\pm \mathrm{SD}$ of the crosscorrelation coefficient of the waveforms between with and without insoles was $R=0.89 \pm 0.09$. For the shear force, it was $R=0.86 \pm 0.17$. This indicates high correlation. And for the stance time, the mean \pm SD for (a) without insoles was $0.68 \pm 0.06$ (s) and that for (d) with insoles was $0.69 \pm 0.05$ (s). There were no significant differences $(p=0.69)$. These show that the insoles did not largely alter the gait. For the VAS scores that indicates the comfortableness, the mean \pm SD for (a) was $3.3 \pm 2.6$ $(\mathrm{cm})$ and that for $(\mathrm{d})$ was $6.8 \pm 1.5(\mathrm{~cm})$. There was significant difference between (a) and (d). The subjects felt more comfortable with insoles than barefoot.

\section{Evaluation of the assessment ability of footwear effect on the plantar}

We evaluated the ability of the system to assess the effect of the footwear on the plantar by measuring multiple subjects and various footwear.

\section{Differences between simulated barefoot walking and walking with the subject wearing their usual footwear, obtained using multiple subjects}

To evaluate the effects of the various footwear on the plantar, 10 subjects walked using their own footwear. Because their usual shoes were different, we could obtain data of a variety of shoes. They could also walk naturally without requiring a long time to get used to the footwear. Moreover, in many cases, the footwear was selected by both their preference and the desire to reduce the pressure and shear force. We therefore calculated the ratio of the measured values for (e) their own footwear to those for (a) barefoot.

Considering the weight of the shoes, the sizes of the shoes, and the difference between the shoes size and the plantar size, the mean values of the shear force for five higher subjects and five lower subjects were compared by t-test. The mean values of the peak shear force of 
Table 3 Mean peak pressure and peak shear force with their own shoes

\begin{tabular}{|c|c|c|c|c|c|c|c|c|c|c|c|c|}
\hline \multicolumn{4}{|c|}{ Usual wearing shoes } & \multicolumn{4}{|c|}{ Shoes vs. barefoot } & \multirow[t]{2}{*}{ Shoes type } & \multirow[t]{2}{*}{ Insoles } & \multirow{2}{*}{$\begin{array}{l}\text { Heel } \\
\text { height }\end{array}$} & \multirow{2}{*}{$\begin{array}{l}\text { Heel } \\
\text { hardness }\end{array}$} & \multirow[t]{2}{*}{ Callus } \\
\hline$\overline{\mathrm{PPH}}$ & PPP & PS & ST & $\overline{\mathrm{PPH}}$ & PPP & PS & $\overline{\text { ST }}$ & & & & & \\
\hline 3.79 & 5.53 & 3.22 & 0.68 & 88 & 54 & 175 & 100 & high-heeled & $\mathrm{n}$ & 7.5 & 40 & $y$ \\
\hline 3.04 & 5.42 & 2.27 & 0.62 & 69 & 101 & 102 & 105 & high-heeled & $n$ & 4.5 & 45 & $n$ \\
\hline 6.41 & 6.38 & 1.66 & 0.65 & 71 & 76 & 67 & 102 & high-heeled & $n$ & 4.5 & 50 & $n$ \\
\hline 3.41 & 5.13 & 2.02 & 0.71 & 90 & 139 & 164 & 101 & high-heeled & $n$ & 4 & 20 & $\mathrm{n}$ \\
\hline 4.29 & 5.80 & 2.58 & 0.69 & 128 & 64 & 62 & 106 & casual & y & 3.5 & 30 & $n$ \\
\hline 3.74 & 4.76 & 2.53 & 0.73 & 82 & 69 & 63 & 103 & casual & y & 3 & 45 & $n$ \\
\hline 6.65 & 6.30 & 2.57 & 0.74 & 94 & 103 & 68 & 107 & boots & $y$ & 3 & 80 & $y$ \\
\hline 3.88 & 10.43 & 1.68 & 0.67 & 161 & 150 & 78 & 100 & leather & $\mathrm{n}$ & 2 & 60 & $n$ \\
\hline 10.42 & 6.28 & 4.13 & 0.65 & 184 & 49 & 131 & 94 & sneakers & $y^{*}$ & 1 & 75 & y \\
\hline 2.37 & 3.36 & 1.47 & 0.75 & 67 & 65 & 77 & 93 & sandals & $n$ & 0 & 40 & $\mathrm{n}$ \\
\hline
\end{tabular}

Peak Pressure at Heal strike $\left[\mathrm{kgf} / \mathrm{cm}^{\wedge} 2\right](\mathrm{PPH})$.

Peak Shear force [kgf] (PS).

Shoes vs. barefoot: Shoes / Barefoot $\times 100$ (\%).

Peak Pressure at Push off $\left[\mathrm{kgf} / \mathrm{cm}^{\wedge} 2\right]$ (PPP).

Stance Time [s] (ST).

*the subject wearing sneakers used special insoles.

subjects with and without callus, which seemed to be related to the usually worn shoes, were compared by t-test.

Table 3 lists the ratio of (a) simulated barefoot to (e) wearing usual shoes for each subject.

Although there was no subject whose peak pressure at heel strike, peak pressure at push off, and peak shear force all increased, there were three subjects for whom they decreased. The peak pressure at heel strike increased for three subjects, the peak pressure at push off increased for four subjects, and the peak shear force increased for four subjects.

Under condition (a) barefoot, the mean $\pm \mathrm{SD}$ of the shear forces for the subject with callus was $2.59 \pm 1.08$ (kgf) and that for the subject without callus was $2.67 \pm$ 0.99 (kgf). There was no significant difference $(\mathrm{p}=0.66)$. Under condition (e) wearing usual shoes, the mean \pm SD of the shear forces for the subject with callus was significantly higher than that for the subject without callus $(3.31 \pm 0.78$ (kgf) versus $2.03 \pm 0.42(\mathrm{kgf}), \mathrm{p}=0.01)$.

Regarding the weight of the shoes, sizes of the shoes, and difference between the shoe size and plantar size, the mean values of the shear forces for higher subjects and those for lower subjects were compared. There were no significant differences between two groups.

For the stance time, the mean \pm SD for (a) was 0.68 \pm 0.06 (s) and that for (e) was $0.69 \pm 0.04$ (s). There was no significant difference $(\mathrm{p}=0.51)$. For the VAS scores, the mean $\pm \mathrm{SD}$ for (a) barefoot was $3.3 \pm 2.6$ $(\mathrm{cm})$ and that for (e) usual shoes was $5.4 \pm 1.2(\mathrm{~cm})$. Although the scores for (e) usual shoes were higher than those for (a) barefoot, there was no significant difference $(\mathrm{p}=0.07)$.

\section{Differences between the measured values for different footwear of a subject with callus}

To evaluate the effectiveness of intervention by footwear, we evaluated the difference between the effects of the footwear on the plantar for a subject with callus. The selected subject was the one with the highest peak shear force for (a) simulated barefoot for subjects with callus.

The subject walked using (a) simulated barefoot, usual shoes (boots), leather shoes, and therapeutic shoes, respectively. By comparing the measured values of (a) barefoot with those of the different footwear, we evaluated the system by examining whether the effect of reducing the pressure and shear force could be measured. By comparing when the usual shoes (boots) and the other footwear were worn, we examined whether their differences caused by the characteristics of the footwear, such as weight, hardness of the heel region and shapes, could be measured. Leather shoes were selected for two major reasons. One was that leather shoes are commonly used. The other is that they are different from boots and therapeutic shoes in terms of the shoelace, weight, hardness of the heel region, etc. Therapeutic shoes used in this evaluation were for prevention of foot ulceration. Generally, the shoes are used with custom-made insoles designed to closely fit the plantar of the patient. Because the subject did not have foot deformities, we used commercially available insoles for the measurement instead of custom-made insoles.

We used a t-test to compare the peak pressure, peak shear force, and stance time. We also calculated the crosscorrelation coefficient between the waveforms. These are performed between (a) and other footwear and also between usual shoes (boots) and other footwear. 

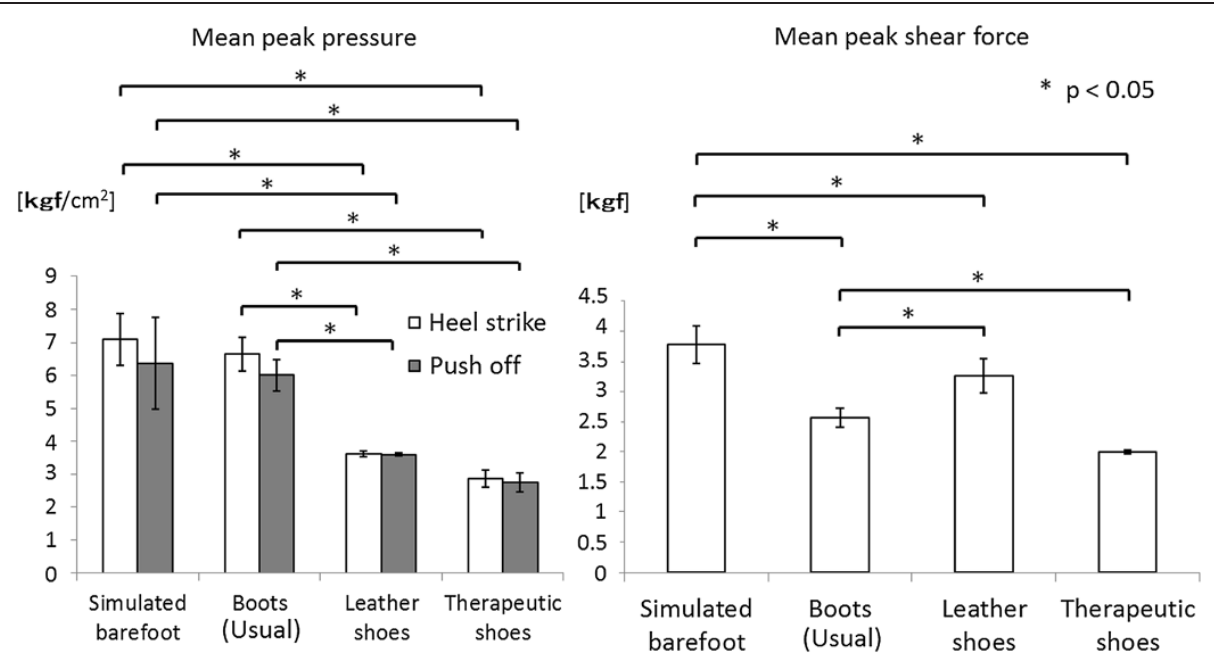

Figure 8 Mean peak pressure and peak shear force with various footwear (same subject).

Figure 8 shows the mean \pm SD of the measured values when the same subject with callus walked using three types of footwear.

Compared to (a) barefoot, leather and therapeutic shoes significantly reduced the peak pressures at heel strike and push off ( $\mathrm{p}<0.001$ for each case). The crosscorrelation coefficients of the waveforms of the peak pressure were $R=0.86$ and $R=0.82$, and those of the shear force were $R=0.92$ and $R=0.83$. The usual shoes (boots) did not significantly reduce the peak pressures at heel strike $(\mathrm{p}=0.10)$ and push off $(\mathrm{p}=0.61)$ but significantly reduced the peak shear force $(p<0.001)$. The cross-correlation coefficient of the waveforms of the peak pressure was $R=0.78$ and that of the shear force was $\mathrm{R}=0.84$.

Compared to those for usual shoes (boots), the peak pressures at heel strike and push off for leather and therapeutic shoes were significantly lower $(\mathrm{p}<0.001$ for each case). The peak shear force for leather shoes was significantly higher and that for therapeutic shoes was significantly lower than that for boots $(\mathrm{p}<0.001$ for each case). The cross-correlation coefficients of the waveforms of the peak pressure were $R=0.96$ and $R=0.93$, and those of the shear force were $R=0.93$ and $R=0.73$. Peak shear force of therapeutic shoes was $2.00 \pm 0.03$ (kgf). The value is almost the same as the peak shear of subjects without callus with their usual footwear $(2.03 \pm$ 0.42 (kgf)).

The stance time for (a) barefoot, boots, leather shoes, and therapeutic shoes were $0.68 \pm 0.02,0.74 \pm 0.02,0.75 \pm$ 0.02 , and $0.76 \pm 0.02(\mathrm{~s})$, respectively. Compared to those for (a) barefoot, the stance time for other footwear were significantly longer ( $\mathrm{p}<0.001$ for each case).

\section{Results}

1) Development of a system for measuring the direct in shoe pressure and shear on the plantar during gait

2) Verification of the implemented system The values measured during a trial were stable and better than those obtained by the previous system (reliability)

The values measured during a trial and between trials were similar to those obtained by the previous system (criteria-related validity)

The values measured between trials (reliability and criteria-related validity)

Could detect the difference between subjects (content validity)

Confirmed the decrease of the load using the insoles, which seemed to reduce the pressure and shear (content validity)

3) Evaluation of the assessment ability of footwear effect on the plantar

Differences between simulated barefoot walking and walking with the subject wearing their usual footwear, obtained using multiple subjects Differences between the measured values for different footwear of a subject with callus

\section{Discussion}

Only the peak shear force obtained by the system was significantly higher compared to that obtained by the previous system. Regarding variations in the shear force, the peak shear forces for (c) on the foot and (b) previous system had a mean value and SD of 0.35 and $0.23\left(\mathrm{kgf} / \mathrm{cm}^{2}\right)$, respectively. The mean of the SDs for 
(c) on the foot was significantly higher than that for (b) previous system $(\mathrm{p}=0.03)$. However, the mean of the measured values for (c) on the foot was $1.93 \pm 0.84$ $\left(\mathrm{kgf} / \mathrm{cm}^{2}\right)$, which was significantly higher than that for (b) previous system, which was $1.31 \pm 0.86\left(\mathrm{kgf} / \mathrm{cm}^{2}\right)$ $(\mathrm{p}<0.001)$. The coefficient of variation $\left(\mathrm{SD} / \mathrm{Mean}{ }^{* 100)}\right.$ for (c) on the foot was $23.8 \pm 18.24\left(\mathrm{kgf} / \mathrm{cm}^{2}\right)$ and that for (b) previous system was $21.59 \pm 10.62\left(\mathrm{kgf} / \mathrm{cm}^{2}\right)$. The absence of significant difference between the results of the paired t-tests $(\mathrm{p}=0.74)$ indicated that the new system was as reliable as the previous one. The new system represents progress in terms of the appulse of the shear force sensor because the previous one might have misesteemed the shear force on the first and second metatarsal heads as the risk regions.

Furthermore, the values measured by the previous system were affected by the 7-mm-thick specialized insoles. Actually, the pressure and shear force for (b) previous system were significantly lower than those for (a) new system. In this respect as well, the new system represented progress because the previous one might have underestimated the risk on the local regions.

Needless to add, the possibility of measuring the in-shoe pressure and shear force directly using various footwear represented great progress. A typical indication of the progress is the absence of significant difference between the peak shear forces of subjects with and without callus when walking barefoot, although subjects with callus exhibited significantly higher peak shear forces than subjects without callus when wearing their own shoes. Additionally, by changing the footwear, the subject with callus reduced the peak shear to almost the same value of subjects without callus.

Although in many cases the footwear reduced the pressure and shear force, as indicated in Table 3 and Figure 8, this was not always the case. Regarding the characteristics of the shoes, for example, as shown in Figure 8, there was no significant difference between the peak pressures for boots and barefoot. Because the weight of each of the boots was $800 \mathrm{~g}$ and the hardness of the heels was 80 durometers, the reduction in the pressure may be small. The corresponding properties for leather shoes and therapeutic shoes were $300 \mathrm{~g}$ and 45 durometers, and $500 \mathrm{~g}$ and 20 durometers, respectively. However, boots produced a significantly higher peak pressure and lower shear force than leather shoes. One possible reason for this was that the boots had shoelace whereas the leather shoes did not. Because the therapeutic shoes also had shoelace, it is considered that fixing the instep with a shoelace would be effective for reducing the shear force.

The effect of fixing the instep with a shoelace on the shear force for high-heeled shoes can also be observed from Table 3. The increase of shear force for highheeled shoes has been reported [20]. In the present study, however, the shear force was reduced for one of the subjects who wore high-heeled shoes. One possible reason for this is that the high-heeled shoes had straps and the fixing of the instep by the straps might have reduced the shear force. The ratio of the shear force for shoes to that for barefoot for the other subject that wore high-heeled shoes with straps was 102\%. Because the ratios for the other two subjects that wore high-heeled shoes without straps were $175 \%$ and $168 \%$, respectively, fixing of the instep may be effective for reducing the shear force even when high-heeled shoes are worn. There were no significant differences between the peak shear forces for the four subjects that wore high-heeled shoes and the other six subjects $(p=0.72)$, nor between those of the group of five subjects with heel heights greater than $3.5 \mathrm{~cm}$ and the five subjects with heel heights lower than $3 \mathrm{~cm}(\mathrm{p}=0.81)$. This suggests that a high peak shear was not simply produced by heel height.

In Table 3, one of the subjects with insoles exhibited increases in the peak shear force and peak pressure at heel strike. The insoles of the subject had only a heel region and no toe region and were made from hard material. This type of insoles was used for reasons of comfortableness. Because of the hardness of the heel region, the peak pressure at heel strike increased and the peak pressure at push off decreased [29]. The increase in the peak shear force was observed only for this footwear and not for the high-heeled shoes. The insoles were also expected to reduce the pressure and shear force. It increases the comfortableness and reduces the pressure and shear force and should therefore be recommended. It may also produce high adherence. This, however, also suggests the risk of selecting the insole based on comfortableness only.

In this study, the changes in gait were estimated by the correlation between the waveforms and the stance time. The degree of gait change could be estimated by this method and the results indicated that footwear largely affected gait change. Knowledge of this gait change is important to the development of footwear. This system can be used together with accelerometers or a motion capture system to determine the relationship between gait features and the in-shoe pressure and shear force directly on the plantar.

\section{Conclusions}

In this study, we developed an easy-to-use system to measure directly the in-shoe plantar pressure and shear force simultaneously during gait with various footwear. Comparing the system with our previous system, which is for measuring the plantar pressure and shear inside footwear using specialized shoes and insoles, the new system has reliability as same as the 
previous system. And the system has high validity for measuring the pressure and shear force on high risk regions.

Furthermore, by using the system for assessing the effect of footwear, we found four suggestions as the following; a) Wearing of footwear does not always reduce both the pressure and shear. b) Fixing the instep with shoelace or straps is effective in the reduction of shear force. c) People with callus show higher peak shear force than people without callus when they walk with their usual regular shoes. d) People with callus can reduce the shear force by changing footwear.

The subjects of this study were healthy adults. Although the relationship between the shear force when wearing the usual footwear and callus formation was suggested, the threshold should be investigated in a future study. Because the safety of this system in clinical settings has already been demonstrated, the system can be used for the evaluation of footwear for prevention and therapy, for example, the study of footwear for the prevention of callus formation in diabetic patients and the evaluation of the risk of fall during gait in the case of elderly using their own shoes.

This system may help assessing the effect of the intervention on foot problems such as footwear or instruction of a walk.

\section{Consent}

Written informed consents were obtained from all subjects for publication of this report and accompanying images.

\section{Competing interests}

The authors declare that they have no competing interests.

\section{Authors' contributions}

MT devised the concept of the sensing equipment, analyzed the data, and drafted the manuscript. HN and MT technically constructed the system, carried out the measurement experiments, and HN and TM developed the analysis method of sequential data. MO and $\mathrm{Hs}$ adjusted the experimental plan based on the clinical point of view, and gave the clinical interpretation of the results. TM devised the basic concept of the overall system, drew the design of the study, and approved the final version of the manuscript. All authors read and approved the final manuscript.

\section{Acknowledgements}

Part of this study was supported by the Japan society for the promotion of science grant-in-aid for scientific research (PN: 25540112).

\section{Author details}

'Department of Life Support Technology (Molten), Graduate School of Medicine, The University of Tokyo, Tokyo, Japan. ${ }^{2}$ Department of Advanced Nursing Technology, Graduate School of Medicine, The University of Tokyo, Tokyo, Japan. 'Department of Gerontological Nursing/ Wound Care Management, Graduate School of Medicine, The University of Tokyo, Tokyo, Japan.

Received: 21 January 2014 Accepted: 24 May 2014

Published online: 23 July 2014

\section{References}

1. Michael WW (1996) Clinical gait analysis: a review. Hum Mov Sci 15(3):369-387

2. Bridenbaugh SA, Kressig RW (2011) The role of gait analysis in seniors' mobility and fall prevention. Gerontology 57(3):256-264

3. Wrobel JS, Najafi B (2010) Diabetic foot biomechanics and gait dysfunction. J Diabetes Sci Technol 4(4):833-845

4. Ornetti P, Maillefert JF, Laroche D, Morisset C, Dougados M, Gossec L (2010) Gait analysis as a quantifiable outcome measure in hip or knee osteoarthritis: a systematic review. Joint Bone Spine 77(5):421-425

5. Menant JC, Steele JR, Menz HB, Munro BJ, Lord SR (2008) Optimizing footwear for older people at risk of falls. J Rehabil Res Dev 45(8):1167-1181

6. Healy A, Naemi R, Chockalingam N (2013) The effectiveness of footwear as an intervention to prevent or to reduce biomechanical risk factors associated with diabetic foot ulceration: a systematic review. J Diabetes Complications 27(4):391-400

7. Hinman RS, Bennell KL (2009) Advances in insoles and shoes for knee osteoarthritis. Curr Opin Reumatol 21(2):164-170

8. García-Álvarez Y, Lázaro-Martínez JL, García-Morales E, Cecilia-Matilla A Aragón-Sánchez J, Carabantes-Alarcón D (2013) Morphofunctional characteristics of the foot in patients with diabetes mellitus and diabetic neuropathy. Diabetes Metab Syndr 7(2):78-82

9. Bus SA (2008) Foot structure and footwear prescription in diabetes mellitus. Diabetes Metab Res Rev 24(Suppl 1):S90-S95

10. Wu S, Armstrong DG (2005) Risk assessment of the diabetic foot and wound. Int Wnd J 2(1):17-24

11. Patry J, Belley R, Côté M, Chateau-Degat ML (2013) Plantar pressures, plantar forces, and their influence on the pathogenesis of diabetic foot ulcers: a review. J Am Podiatr Med Assoc 103(4):322-332

12. Boulton AJ (2004) Pressure and the diabetic foot: clinical science and offloading techniques. Am J Surg 187(5A):17S-24S

13. Murray HJ, Young MJ, Hollis S, Boulton AJ (1996) The association between callus formation, high pressures and neuropathy in diabetic foot ulceration. Diabet Med 13(11):979-982

14. Bakker K, Apelqvist J, Schaper NC, International Working Group on Diabetic Foot Editorial Board (2012) Practical guidelines on the management and prevention of the diabetic foot 2011. Diabetes Metab Res Rev 28(Suppl 1):225-231

15. Fernández-Seguín LM, Diaz Mancha JA, Sánchez Rodríguez R, Escamilla Martínez E, Gómez Martín B, Ramos Ortega J (2014) Comparison of plantar pressures and contact area between normal and cavus foot. Gait Posture 39(2):789-792. Epub ahead of print

16. Menz HB, Lord ST, Mclntosh AS (2001) Slip resistance of casual footwear: implications for falls in older adults. Gerontology 47(3):145-149

17. Yavuz M, Erdemir A, Botek G (2007) Peak plantar pressure and shear locations: relevance to diabetic patients. Diabetes Care 30(10):2043-2045

18. Maly MR, Culham EG, Costigan PA (2002) Static and dynamic biomechanics of foot orthoses in people with medial compartment knee osteoarthritis. Clin Biomech 17(8):603-610

19. Lord M, Hosein R (2000) A study of in-shoe plantar shear in patients with diabetic neuropathy. Clin Biomech 15(4):278-283

20. Cong Y, Cheung JT, Leung AK, Zhang M (2011) Effect of heel height on in-shoe localized triaxial stresses. J Biomech 44(12):2267-2272

21. Mori T, Hamatani M, Noguchi H, Oe M, Sanada H (2012) Insole-type simultaneous measurement system of plantar pressure and shear force during gait for diabetic patients. J Robotics Mechatronics 24(5):766-772

22. Mueller MJ, Strube MJ (1996) Generalizability of in-shoe peak pressure measures using the F-scan system. Clin Biomech 11(3):159-164

23. Hsiao H, Guan J, Weatherly M (2002) Accuracy and precision of two in-shoe pressure measurement systems. Ergonomics 45(8):537-555

24. Ashry HR, Lavery LA, Murdoch DP (1997) Effectiveness of diabetic insoles to reduce foot pressures. J Foot Ankle Surg 36(4):268-271

25. Lavery LA, Lanctot DR, Constantinides G (2005) Wear and biomechanical characteristics of a novel shear-reducing insole with implications for high-risk persons with diabetes. Diabetes Technol Ther 7(4):638-646

26. Burnfield JM, Few CD, Mohamed OS, Perry J (2004) The influence of walking speed and footwear on plantar pressures in older adults. Clin Biomech 19(1):78-84

27. Bus SA, de Lange A (2005) A comparison of the 1-step, 2-step, and 3-step protocols for obtaining barefoot plantar pressure data in the diabetic neuropathic foot. Clin Biomech 20(9):892-899 
28. Mills K, Blanch P, Vicenzino B (2010) Identifying clinically meaningful tools for measuring comfort perception of footwear. Med Sci Sports Exerc 42(10):1966-1971

29. Lane TJ, Landorf KB, Bonanno DR (2014) Effects of shoe sole hardness on plantar pressure and comfort in older people with forefoot pain. Gait Posture 39(1):247-251

doi:10.1186/s40648-014-0004-9

Cite this article as: Takano et al:: Development and evaluation of a

system to assess the effect of footwear on the in shoe plantar pressure and shear during gait. ROBOMECH Journal 2014 1:4.

Submit your manuscript to a SpringerOpen ${ }^{\circ}$ journal and benefit from:

- Convenient online submission

- Rigorous peer review

- Immediate publication on acceptance

- Open access: articles freely available online

- High visibility within the field

- Retaining the copyright to your article 